\title{
Osteoarthritic changes rather than age predict outcome following arthroscopic treatment of femoroacetabular impingement in middle-aged patients
}

\author{
Simon Jakob Herrmann*, Manuel Bernauer, Benjamin Erdle, Norbert Paul Südkamp, Peter Helwig \\ and Oliver Hauschild
}

\begin{abstract}
Background: Our purpose was to evaluate outcome following arthroscopic treatment of femoroacetabular impingement (FAl) in middle-aged patients and to define risk factors for conversion to total hip arthroplasty (THA).

Methods: This was a retrospective case series of 79 consecutive patients (40 to 65 years) undergoing arthroscopic treatment of FAl (follow-up $\geq 12$ months). Outcome at follow-up was assessed using Hip outcome score (HOS). Alpha angle, Kellgren Lawrence grade (K-L grade), joint space width (JS), lateral center edge (LCE) angle, caput-collum-diaphysis (CCD) angle and acetabular index (AI) were analysed retrospectively. THA group and Non-THA group were compared.

Results: Seventy-nine patients (mean age 48.6 years, mean follow-up 32 months) were included. 18 patients (22.8\%) were converted to THA. Mean HOS score in the Non-THA group at time point of follow-up was 80.2. Non-THA group and THA group showed no significant differences for mean age (48.2 years vs. 49.9 years, $p=0.278$ ), alpha angel $(p=0.541), \operatorname{LCE}(p=0.294), C C D(p=0.101)$ and $\mathrm{Al}(p=0.661)$ in contrast to differences for JS $(p=<0.001)$ and $\mathrm{K}-\mathrm{L}$ grade $(p=<0.001)$. Risk of conversion to THA was higher for patients with K-L grade $3(p=0.003)$ or joint space less or equal $2 \mathrm{~mm}(p=0.001)$.
\end{abstract}

Conclusions: One fifth of the middle-aged patients required early conversion to THA. Advanced JS narrowing and K-L grade rather than age alone can be considered as risk factor for conversion to THA.

Keywords: Femoroacetabular impingement, Hip arthroscopy, Alpha angle, Hip outcome score

\section{Background}

The concept of FAI has become of growing clinical importance and as a result the number of arthroscopic interventions to the hip joint is rising [1]. FAI syndrome was described as a subtle abnormal bone morphology at the femoral head neck junction or the acetabulum causing an intracapsular bony conflict between proximal femur and acetabular rim under motion within the physiological range [2, 3]. This conflict leads to labral tears and abrasion or avulsion of the the cartilage at the

\footnotetext{
* Correspondence: simon.herrmann@uniklinik-freiburg.de

Department for Orthopedic and Trauma Surgery, Freiburg University Medical Center, Hugstetterstr. 55, 79106 Freiburg, Germany
}

acetabular rim and there is evidence that FAI may lead to osteoarthritis (OA) in the long term [2].

Within the last decade, arthroscopic intervention has proven to be an effective treatment for FAI $[4,5]$. Arthroscopy allows the surgeon to address the pathological morphology at the femoral head neck junction and the acetabular rim and thereby restore the functional integrity of the hip joint and possibly prevent further degenerative damage to the joint. Prospective studies have shown improving range of motion, pain relief, scores after arthroscopic treatment in the younger population $[4,6,7]$. 
However, little is known about the outcome in middleaged patients. It can be assumed that in these patients the conflict between proximal femur and acetabular rim has existed for a longer time and advanced degenerative changes of the cartilage and labrum may already be present at time of intervention [8,9]. As a consequence this might jeopardize the effects of any intervention aiming at restoration of regular joint anatomy. Some of these patients may therefore not benefit from hip preserving surgery but rather require early conversion to total hip arthroplasty (THA). In an effort to facilitate preoperative decision making it is important to define parameters predicting poor outcome and risk of early conversion to THA [10].

To describe the abnormal head neck offset, the concept of alpha angle was introduced and widely used to define cam impingement [11]. The relation between alpha angle and increased chondral damage and labral injury as well as early progression to THA was described $[12,13]$. However, until today there is no study showing correlation between preoperative alpha angle and outcome after arthroscopic treatment.

The aim of this study was to evaluate the mid term outcome following arthroscopic treatment of FAI in middle-aged patients and to define preoperative prognostic factors for early conversion to THA.

\section{Methods}

This was retrospective case series of consecutive patients undergoing arthroscopic treatment of FAI at our institution between January 2009 and August 2012. The study was approved by the local ethical committee (EK 225/ 13). Minimum follow up time was 12 months. Of the 209 patients operated within this period, 99 met the inclusion criteria, for inclusion and exclusion criteria see Table 1 . The indication for surgery was groin pain in combination with radiographic diagnosis of FAI or labral tear detected in magnetic resonance imaging (MRI).

Three surgeons highly experienced in arthroscopic intervention were involved in the surgical treatment. Arthroscopic intervention was performed with the patient in supine position on a fracture table under general anesthesia and antibiotic prophylaxis with cefuroxime.

Cam impingement was treated by femoral osteochondroplasty. Synovial debridement was performed in cases with synovitis. Labral tear was treated by partial resection of the labrum or labral repair with suture anchors at the surgeon's discretion. Acetabular overcoverage was treated with acetabular rim trimming and refixation of the labrum. Postoperative partial weight bearing with $20 \mathrm{~kg}$ was indicated for 3 weeks after femoral osteochondroplasty and for 6 weeks after labral repair. Flexion of the hip joint was restricted to $90^{\circ}$ for 3 weeks postoperatively. All patients were referred to outpatient physiotherapy. Indomethacin was administerd for 14 days postoperatively for all patients to prevent heterotopic ossification.

Upon institutional review board approval conventional radiographs were analyzed retrospectively by one observer $(\mathrm{SJH})$. Alpha angle was assessed in cross-table lateral view [11]. Lateral-center-edge (LCE) angle, caput-collumdiaphysis (CCD) angle and acetabular index (AI) were measured in cross-table lateral radiographs [14]. Joint space width (JS) was measured as the narrowest distance between acetabulum and femoral head at three points (superior lateral edge, middle of the sourcil, at the border to the fovea). All measurements were performed using the digital caliper system of OfficePACS system with an accuracy of one decimal place. All radiographs were graded using the Kellgren Lawrence classification [15].

At time point of follow up patients were examined by telephone interview. Further surgery done to the hip in question including conversion to THA and time point of reoperation was recorded. We asked for the subjective change in joint function following intervention on a scale from-100\% (representing maximum of deterioration) to $+100 \%$ (representing maximum of improvement). Level of satisfaction with the surgical intervention and outcome was categorized from 1 (completely unsatisfied) to 4 (completely satisfied). Patient were asked, whether they would retrospectively opt for hip arthroscopy as a treatment option again. Additionally, the German version of the hip outcome score (HOS) and the

Table 1 Inclusion and exclusion criteria

\begin{tabular}{|c|c|}
\hline Inclusion criteria & Exclusion criteria \\
\hline $\begin{array}{l}\text { Patients undergoing hip arthroscopy for FAI between } 1 / 2009 \text { and } 8 / 2012 \\
\text { Indication for arthroscopic surgery: groin pain, at least on positive clinical test (FABER, FADIR, anterior } \\
\text { impingement test, apprehension test), radiographic signs of FAI (alpha angle }>55^{\circ} \text { or center of femoral } \\
\text { head medial to posterior wall or posterior acetabular wall crosses anterior acetabular wall), labral tear in MRI }\end{array}$ & $\begin{array}{l}\text { Other indication for hip arthroscopy } \\
\text { (trauma, ECF, empyema) }\end{array}$ \\
\hline \multicolumn{2}{|l|}{ Age at time point of surgery between 40 and 65 yrs } \\
\hline Preoperative radiological diagnostic (plain radiograph in anterior posterior or cross table projection) & No preoperative plain radiograph. \\
\hline At least 12 mos post hip arthroscopy & \\
\hline
\end{tabular}


hip outcome score sports subscale (HOSS) were assessed for all patients without conversion to THA.

\section{Statistical analyses}

Survivorship was defined as preservation of the native hip joint until follow-up. Patients who had undergone conversion to THA in the time between index operation and follow-up (THA group) were compared to patients who did not require conversion to THA (Non-THA group). Kolmogorov-Smirnov test confirmed normally distributed outcome data for both groups. A parametric $T$-test for independent samples was used to compare age and radiographic parameters between THA and NonTHA groups.

To determine the effect of osteoarthritic changes on outcome patients were divided in groups with Kellgren Lawrence (K-L) grades 0,1 or 2 and K-L grade 3 and JS $>2 \mathrm{~mm}$ and JS $\leq 2 \mathrm{~mm}$, respectively and frequency tables were analysed using Fisher's and Chi-square tests. Spearman's rho correlation coefficient was used to describe correlations between categorical and continuous, normally distributed data. Pearson correlations were used to detect associations between continuous, normally distributed variables. All reported $p$-values are two tailed with an alpha level of 0.05 indicating significance.

SPSS Version 21 (SPSS Inc, Chicago, IL, USA) was used for all statistical analysis.

\section{Results}

Seventy-nine patients ( $80 \%)$ out of 99 patients fulfilling inclusion criteria were available for follow up interviews. Mean follow up period was 32 months. Mean age at time of index surgery was 48.6 (Table 2).

Eighteen patients $(22.8 \%)$ underwent conversion to THA within the follow up period. Minimum survivorship in the Non-THA group was 5 month and maximum was 45 months. Non-THA group and THA group were comparable with regard to age and all preoperatively assessed angles (Table 3, Fig. 1). However, patients with $\mathrm{K}-\mathrm{L}$ grade of 3 were significantly more likely to require THA following arthroscopic intervention as compared to those with K-L grade 2 or less (66.7 vs. $16.2 \%, P=$ 0.003). The same was true for patients with a JS of $2 \mathrm{~mm}$ or less as compared with JS $>2 \mathrm{~mm}$ (75 vs. $15.9 \%$, $P=0.001)$. Data are summarized in Tables 3 and 4 and Fig. 2.

Table 2 Descriptive statistics

\begin{tabular}{ll}
\hline Follow up period (min 12 mos) & $32.3(\mathrm{SD} \pm 12.9)$ mos \\
Age at time of index surgery & $48.6(\mathrm{SD} \pm 6.1) \mathrm{yrs}$ \\
Conversion to THA & 18 patients (22.8 \%) \\
Survivorship group THA & min 5 mos, max 45 mos \\
\hline
\end{tabular}

THA total hip arthroplasty, yrs years, mos months, $S D$ standard deviation, min minimum, max maximum
Accordingly, $22.8 \%$ of patients from THA group would retrospectively choose for arthroscopic treatment again, as compared to $78.8 \%$ from the Non-THA group $(p<0.001$, Chi Square). However, within the THA group time of survivorship did not significantly correlate with differences between patients who would chose arthroscopic treatment again and the patients who would refuse (rho $=0.335, P=$ 0.174 ). In the THA group we did not see a correlation between the decision in favour of arthroscopic treatment and survivorship (rho $=0.191, P=0.447$ ).

Mean HOS score in the Non-THA group at time point of follow up was $80.2( \pm 16.2)$ and mean HOSS was 63.2 $( \pm 24.8)$. Mean subjective change in function in the NonTHA group was $+54.3 \%( \pm 41.5 \%)$, mean patient related satisfaction $3.2( \pm 0.9)$ (Table 5). HOS did not correlate with any of the preoperatively assessed angles (Pearson's correlation coefficient $-0.04<r<0.21$ ).

We saw two revision hip surgeries because of treatment failure (one open revision surgery and microfracturing, one arthroscopic revision surgery and labral repair) and one surgery because of major complication (1.3\%, osteosynthesis of insidious femoral neck fracture).

\section{Discussion}

The aim of this study was (1) to evaluate the mid-term outcome and patient satisfaction following arthroscopic treatment of FAI in middle-aged patients and (2) to define preoperative radiographic factors predictive for outcome. The most important findings of this study were that (1) age alone should not be seen as contraindication for successful treatment of FAI and reasonable outcome can be expected for the majority of patients and (2) a preoperative K-L grade 3 and/or a joint space width of less than $2 \mathrm{~mm}$ will be associated with high rates of early conversion to THA and poor patient satisfaction with arthroscopic intervention.

Appreciation of the limitations of this study is warranted. It is a strength of this study that it is based on a consecutive series of patients. However, this is an analysis of retrospectively collected data with inherent limitations typical of retrospective studies. First and foremost, there was no control group and preoperative scores were not available. Yet, THA and non-THA groups were comparable with regard to demographic parameters. Owing to the lack of preoperative scores we cannot exclude a difference in preoperative functional impairment inbetween groups. In this regard our study can not give an answer to the question whether there is a threshold in preoperative functional scoring associated with an elevated risk for early conversion to THA.

The validity of the HOS may have been compromised by the assessment in telephone interview. Nevertheless, the outcome measurements were comparable to those reported in the literature. 
Table 3 Mean values of patient characteristics and T-test comparison of non-THA and THA group

\begin{tabular}{|c|c|c|c|c|c|}
\hline & & All & Group non-THA & Group THA & $P$ value \\
\hline Number of patients & & 79 & $61(77.2 \%)$ & $18(22.8 \%)$ & \\
\hline Male/female & & $49 / 30$ & $38 / 23$ & $11 / 7$ & \\
\hline Age at surgery in years & (SD) & $48.6( \pm 3.3)$ & $48.2( \pm 6.2)$ & $49.9( \pm 5.6)$ & 0.278 \\
\hline K-L grade & & $1.45( \pm 0.8)$ & $1.27( \pm 0.8)$ & $2.12( \pm 0.8)$ & $<0.001^{*}$ \\
\hline $\mathrm{JS}$ in $\mathrm{mm}$ & & $3.3( \pm 1.0)$ & $3.5( \pm 0.8)$ & $2.3(1.1)$ & $<0.001^{*}$ \\
\hline Alpha angle $\left(^{\circ}\right)$ & & $67( \pm 13)$ & $67.5( \pm 12.6)$ & $64.7( \pm 15)$ & 0.541 \\
\hline LCE angle $\left(^{\circ}\right)$ & & $32( \pm 7.5)$ & $32( \pm 7.3)$ & $31.5( \pm 8.7)$ & 0.77 \\
\hline CCD angle $\left(^{\circ}\right)$ & & $130.9( \pm 5.4)$ & $131( \pm 5.6)$ & $130.8( \pm 5.1)$ & 0.92 \\
\hline $\mathrm{Al}\left({ }^{\circ}\right)$ & & $6.6( \pm 5.6)$ & $6.3( \pm 5.6)$ & $7.4( \pm 5.5)$ & 0.511 \\
\hline
\end{tabular}

THA total hip arthroplasty, $K$-L grade Kellgren Lawrence grade, JS joint space width, $L C E$ lateral center edge angle, $C C D$ caput-collum-diaphysis angle, $A I$ acetabular index, $S D$ standard deviation

"Significant at 0.05 level

${ }^{\mathrm{a}}$ T-test

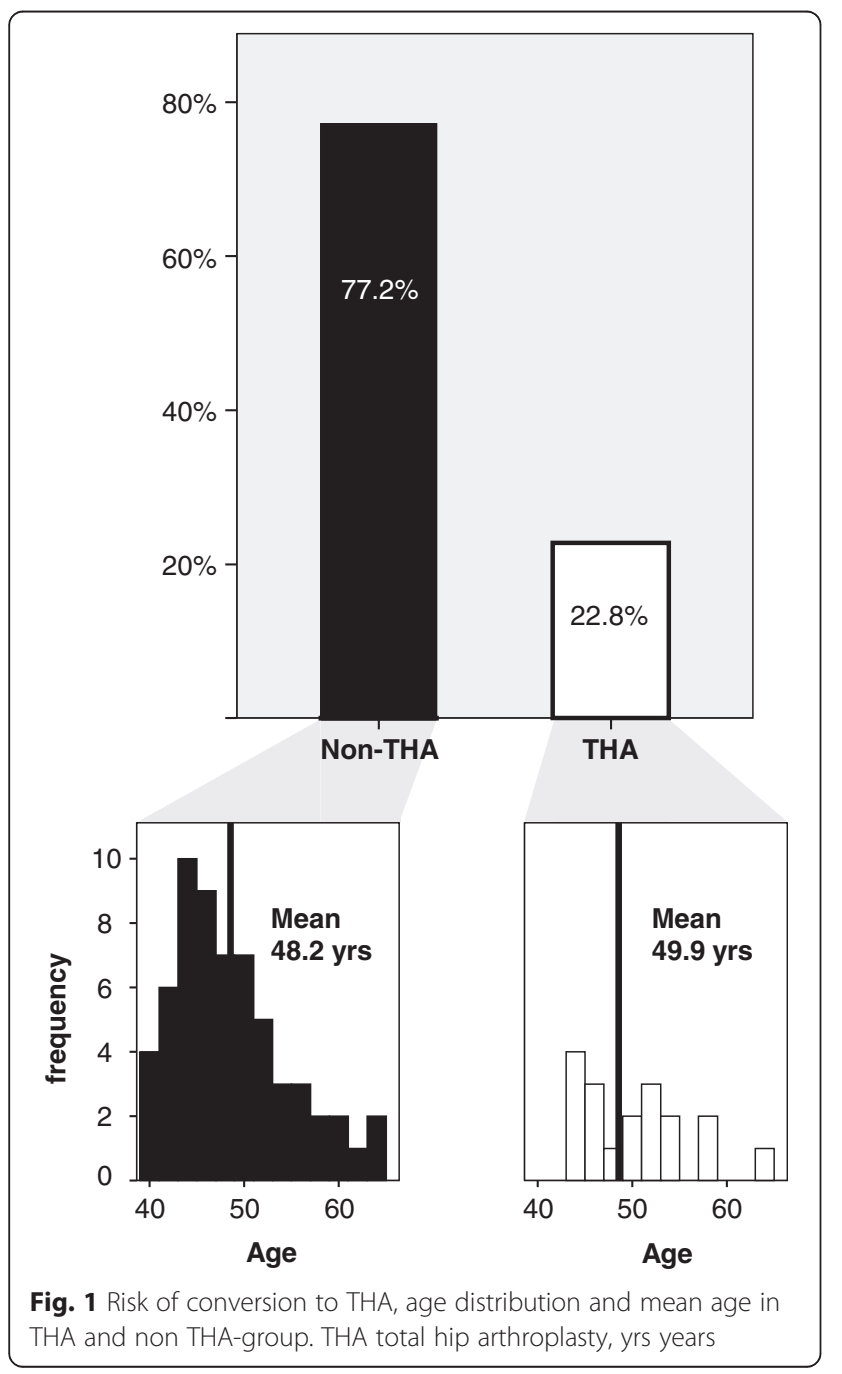

There was a limited number of patients included in the study, ending up with only 18 patients in the THA group. Comparable to other studies reporting on arthroscopic treatment of FAI, the follow up time was limited and the follow up period ranged from 12 to 55 months. Still, the mean follow up of the present study was almost 3 years with a maximum of 45 months. Thus, we can only report on midterm outcome, the number of conversions to THA in the long run cannot be derived from our data and remains unclear. Owing to the retrospective nature of the study follow-up varied widely from 12 to 45 months. This should be kept in mind when interpreting the results. Moreover, follow-up was significantly shorter in the non-THA as compared to the THA group (30.9 vs. 39.0 months, $P=0.02$ ). This might bias the results in favour of the non-THA group. It is not unreasonable to assume, that the rate of conversions to THA would have increased with equal follow-up. Given the strong association of Kellgren-Lawrence grade and joint

Table 4 Increasing percentage of patients requiring conversion to THA in dependence of preoperative advanced joint space narrowing and high $\mathrm{K}-\mathrm{L}$ grade

\begin{tabular}{llll}
\hline & & Non-THA & THA \\
& & $N$ & $N(\%)$ \\
\hline JS & $>4$ & 20 & $1(4.8)$ \\
in mm & $>2, \leq 4$ & 38 & $10(20.8)$ \\
& $\leq 2$ & 2 & $6(75)$ \\
& $>2$ & 58 & $11(15.9)$ \\
K-L & 0 & 8 & $0(0)$ \\
grade & 1 & 31 & $4(11.4)$ \\
& 2 & 18 & $7(28)$ \\
& 3 & 3 & $6(66.7)$ \\
& Group & 57 & $11(16.2)$ \\
& $0,1,2$ & & \\
\hline
\end{tabular}

THA total hip arthroplasty, JS Joint space width, $K-L$ Kellgren Lawrence 


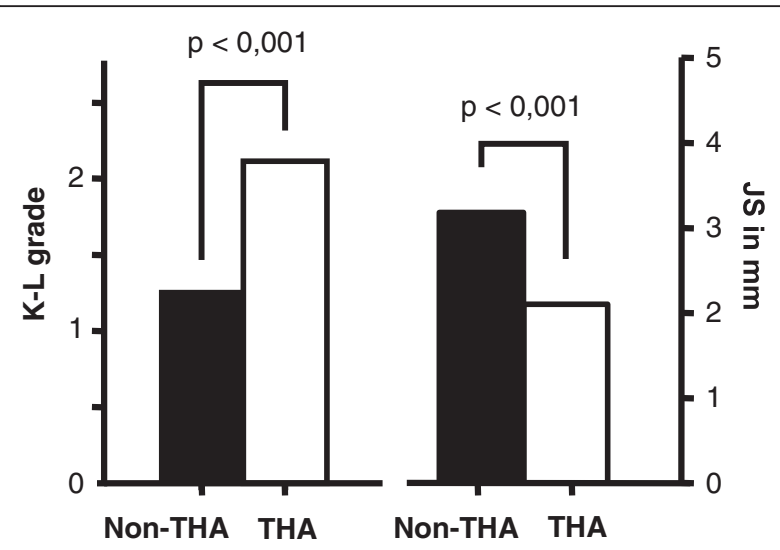

Fig. 2 Mean Kellgren Lawrence grade and joint space width for non-THA and THA group. T-test reveals significant differences. THA total hip arthroplasty, K-L grade Kellgren Lawrence grade, JS Joint space width

space narrowing found in the present analyses we, however, hypothesize that patients with advanced degenerative changes would be most likely to fall from the non-THA into the THA group rather enhancing than jeopardizing the robustness of our results. Yet, in the end this remains speculative.

Finally, 20 patients eligible for inclusion in the study were unavailable for follow-up. Even though only three patients specifically declined participation in the study, a potential bias introduced by loss to follow-up cannot fully be excluded. A follow-up rate of $80 \%$ as achieved in the present study is, nevertheless, generally considered acceptable for retrospective analyses [16].

Arthroscopic treatment of FAI has proven to show significant improvement in outcome scores and effective pain relief in a young, active population [6, 17]. However, little is known about outcome in the older population [18]. In our study, most of the patients showed at least slight radiological signs of OA of the hip (Table 4). Not surprisingly, $22.8 \%$ of the patients included into the study required conversion to THA during follow up period, which is more than it was described for younger populations [4]. This reflects the advanced damage to the hip joint in an older population with FAI leading to failure after arthroscopic treatment [9]. We found that in our study population joint space equal or less $2 \mathrm{~mm}$

Table 5 Outcome scores and subjective outcome parameters

\begin{tabular}{ll}
\hline Characteristic & Mean (SD) \\
\hline HOS & $80.2( \pm 16.2)$ \\
HOS Sport & $63.2( \pm 24.8)$ \\
Subjective change in function & $+54.3 \%( \pm 41.5)$ \\
$(-100 \%$ to $+100 \%)$ & \\
Patient related satisfaction & $3.2( \pm 0.9)$ \\
1 (completely unsatisfied) to 4 (completely satisfied) & \\
\hline
\end{tabular}

HOS Hip outcome score, SD standard deviation and K-L grade more than 2 were strong predictive factors for conversion to THA. This is consistent with a study by Philippon et al., first defining joint space narrowing $2 \mathrm{~mm}$ or less and K-L grade 3 or 4 as a predictor of conversion to THA in 80 and $73 \%$ of the cases [10].

Surprisingly we did not see a correlation between age and risk of conversion to THA for patients between 40 and 65 years of age. The mean age in the THA and the Non-THA groups were comparable. In contrast to our study group of middle-aged patients risk for early conversion to THA reported in the literature for adolescent or young patients without signs of advanced OA effectively approaches zero [4, 7]. However, in our analysis age was not a predictive factor for conversion to THA. Taking into account our results, advanced age itself should not be considered as a contraindication for arthroscopic treatment in patients with FAI.

Early conversion to THA represents, that some patients did not benefit from arthroscopic treatment. However, survivorship within these patients ranged between 5 and 45 months. One could argue, that arthroscopic treatment may have been effective in delaying time to THA at least for some patients. After all, more than one quarter of patients with conversion to THA expressed that they would opt for arthroscopic intervention again. The individual decision did, however, not correlate with time of survivorship. There is still considerable debate on effectiveness of arthroscopic treatment of OA of the hip. Some authors report good outcome after arthroscopic debridement and lavage whereas others see poor results [19-22]. However, one should keep in mind, that outcomes after arthroscopic lavage or arthroscopic debridement for OA of the knee were no better than those after a placebo procedure [23].

The HOS was introduced and validated to measure outcome after treatment of FAI [24]. Until today the numbers of publications measuring functional outcome using $\mathrm{HOS}$ is limited $[5,6,25]$. We reported satisfactory outcome evaluated in HOS and HOSS in the Non-THA group. HOS showed strong correlations with subjective satisfaction (data not shown). In a recent study of 52 patients with a slightly younger study population (median age 42 years) comparable outcome was reported [25]. However, several studies reported superior outcome scores compared to ours in in the young and active population $[6,17]$. Taking into consideration the predictive factors joint space and K-L grade as discussed above, early conversion to THA can be avoided and good outcome can be expected even in mature patients.

Besides clinical tests impingement syndrome is usually diagnosed by defining the pathological cam or pincer morphology in conventional radiographs using alpha angle, LCE or AI $[11,26]$. The alpha angle was introduced to describe the abnormal head neck offset and is used to define cam impingement when exceeding $55^{\circ}$. 
Several recent studies indicate that this border is rather arbitrary [27-29]. In a recent CT-study 36 out of 100 asymptomatic subjects were found to present with alpha angle more than $55^{\circ}$ [27-29]. Mean alpha angle in our symptomatic study population was $67^{\circ}$. Neither could we detect a significant difference in alpha angle between the THA group and the Non-THA group nor was the alpha angle correlated with outcome measured by HOS in the Non-THA group. Alpha angle has been shown to be correlated with age, labral tears and chondral defects $[28,30,31]$. However, our data demonstrate that the outcome after hip athroscopy could not be predicted by preoperative alpha angle. The pathological hip morphology in FAI is too subtle and complex to be described by one single parameter in plain radiographs $[29,31]$. Since clinical tests for FAI have shown to be of limited diagnostic value the diagnosis of FAI based on physical examination and plain radiographs remains challenging [32-34]. In an effort to improve preoperative diagnostic accuracy of FAI and possible additional pathologies future analyses aiming at diagnostic value of contrastenhanced MRI and quantitative assessment of cartilage composition are warranted. Further studies to identify risk factors for treatment failure and early conversion to THA in mature patients are needed.

\section{Conclusion}

Our data show that advanced joint space narrowing and K-L grade should be considered as contraindication for arthroscopic treatment. Taking into account these contraindiacations good mid-term outcome can be expected in middle-aged patients.

\section{Additional files}

Additional file 1: Telephone interview, template for telephone interview in german. (PDF 65 kb)

Additional file 2: Dataset, dataset in tabular form. (PDF $39 \mathrm{~kb}$ )

\section{Abbreviations}

Al, acetabular index; CCD, caput-collum-diaphysis; FABER, flexion, abduction, external rotation; FADIR, flexion, adduction, internal rotation; FAl, femoroacetabular impingement; HOS, hip outcome score; HOSS, hip outcome score sports; JS, joint space width; K-L grade, kellgren lawrence grade; LCE, lateral center edge; max, maximum min: minimum; mos, Months; MRl, magnetic resonance imaging; OA, osteoarthritis; SD, standard deviation; THA: THA; Yrs: years

\section{Availability of data and materials}

The original template for telephone interview (in german) and dataset on which the conclusions of the manuscript rely are presented as Additional files 1 and 2 .

\section{Authors' contributions}

$\mathrm{SJH}$ and $\mathrm{OH}$ conceived the study. All authors were involved in data acquisition and collection. SJH and $\mathrm{OH}$ had main responsibility for manuscript writing. SJH, $\mathrm{BE}, \mathrm{MB}$ and $\mathrm{OH}$ participated in the statistical analyses. $\mathrm{BE}$ helped with the figures. NPS conceived of the study and coordination and helped to draft the manuscript. All authors were involved in interpretation of the data. All authors were involved in drafting the article and approved the final version of the article.

\section{Competing interests}

None of the authors have any competing interests. The authors do not have any commercial or collaborative relationships that could be construed as biased or inappropriate. The decision to submit the paper for publication was not influenced by any funding body.

\section{Consent for publication}

Not applicable.

Ethics approval and consent to participate

Ethical approval was obtained from University of Freiburg Ethics Committee (reference number 225/13).

Received: 20 February 2016 Accepted: 31 May 2016

Published online: 08 June 2016

\section{References}

1. Colvin AC, Harrast J, Harner C. Trends in hip arthroscopy. J Bone Joint Surg Am. 2012;94:e23. doi:10.2106/JBJS.J.01886.

2. Ganz R, Parvizi J, Beck M, Leunig M, Notzli H, Siebenrock KA. Femoroacetabular impingement: a cause for osteoarthritis of the hip. Clin Orthop Relat Res. 2003;112-120. doi:10.1097/01.blo.0000096804.78689.c2.

3. Ito K, Minka 2nd MA, Leunig M, Werlen S, Ganz R. Femoroacetabular impingement and the cam-effect. A MRI-based quantitative anatomical study of the femoral head-neck offset. J Bone Joint Surg (Br). 2001;83:171-6.

4. Byrd JW, Jones KS. Arthroscopic femoroplasty in the management of camtype femoroacetabular impingement. Clin Orthop Relat Res. 2009;467:739-46. doi:10.1007/s11999-008-0659-8.

5. Philippon MJ, Schroder ESBG, Briggs KK. Hip arthroscopy for femoroacetabular impingement in patients aged 50 years or older. Arthroscopy. 2012;28:59-65. doi:10.1016/j.arthro.2011.07.004.

6. Fabricant PD, Heyworth BE, Kelly BT. Hip arthroscopy improves symptoms associated with FAl in selected adolescent athletes. Clin Orthop Relat Res. 2012:470:261-9. doi:10.1007/s11999-011-2015-7.

7. Philippon M, Schenker M, Briggs K, Kuppersmith D. Femoroacetabular impingement in 45 professional athletes: associated pathologies and return to sport following arthroscopic decompression. Knee Surg Sports Traumatol Arthrosc. 2007;15:908-14. doi:10.1007/s00167-007-0332-x.

8. Ganz R, Leunig M, Leunig-Ganz K, Harris WH. The etiology of osteoarthritis of the hip: an integrated mechanical concept. Clin Orthop Relat Res. 2008:466:264-72. doi:10.1007/s11999-007-0060-z.

9. McCarthy JC, Jarrett BT, Ojeifo O, Lee JA, Bragdon CR. What factors influence long-term survivorship after hip arthroscopy? Clin Orthop Relat Res. 2011:469:362-71. doi:10.1007/s11999-010-1559-2.

10. Philippon MJ, Briggs KK, Carlisle JC, Patterson DC. Joint space predicts THA after hip arthroscopy in patients 50 years and older. Clin Orthop Relat Res. 2013:471:2492-6. doi:10.1007/s11999-012-2779-4.

11. Notzli HP, Wyss TF, Stoecklin CH, Schmid MR, Treiber K, Hodler J. The contour of the femoral head-neck junction as a predictor for the risk of anterior impingement. J Bone Joint Surg (Br). 2002;84:556-60.

12. Johnston TL, Schenker ML, Briggs KK, Philippon MJ. Relationship between offset angle alpha and hip chondral injury in femoroacetabular impingement. Arthroscopy. 2008;24:669-75. doi:10.1016/j.arthro.2008.01.010.

13. Nicholls AS, Kiran A, Pollard TC, Hart DJ, Arden CP, Spector T, Gill HS, Murray DW, Carr AJ, Arden NK. The association between hip morphology parameters and nineteen-year risk of end-stage osteoarthritis of the hip: a nested case-control study. Arthritis Rheum. 2011;63:3392-400. doi:10.1002/ art.30523.

14. Werner CM, Ramseier LE, Ruckstuhl T, Stromberg J, Copeland CE, Turen CH, Rufibach K, Bouaicha S. Normal values of Wiberg's lateral center-edge angle and Lequesne's acetabular index-a coxometric update. Skeletal Radiol. 2012:41:1273-8. doi:10.1007/s00256-012-1420-7.

15. Kellgren JH, Lawrence JS. Radiological assessment of osteo-arthrosis. Ann Rheum Dis. 1957;16:494-502.

16. Kristman V, Manno M, Cote P. Loss to follow-up in cohort studies: how much is too much? Eur J Epidemiol. 2004;19:751-60.

17. Philippon MJ, Yen YM, Briggs KK, Kuppersmith DA, Maxwell RB. Early outcomes after hip arthroscopy for femoroacetabular impingement in the athletic adolescent patient: a preliminary report. J Pediatr Orthop. 2008;28:705-10. doi:10.1097/BPO.0b013e318186eb2e. 
18. Javed A, O'Donnell JM. Arthroscopic femoral osteochondroplasty for cam femoroacetabular impingement in patients over 60 years of age. J Bone Joint Surg (Br). 2011;93:326-31. doi:10.1302/0301-620X.93B3.25262.

19. Haviv B, O'Donnell J. The incidence of total hip arthroplasty after hip arthroscopy in osteoarthritic patients. Sports Med Arthrosc Rehabil Ther Technol. 2010;2:18. doi:10.1186/1758-2555-2-18.

20. Kim KC, Hwang DS, Lee CH, Kwon ST. Influence of femoroacetabular impingement on results of hip arthroscopy in patients with early osteoarthritis. Clin Orthop Relat Res. 2007;456:128-32. doi:10.1097/01.blo. 0000246542.49574.2c.

21. Krych AJ, Kuzma SA, Kovachevich R, Hudgens JL, Stuart MJ, Levy BA. Modest mid-term outcomes after isolated arthroscopic debridement of acetabular labral tears. Knee Surg Sports Traumatol Arthrosc. 2014;22:763-7. doi:10.1007/s00167-014-2872-1.

22. Larson CM, Giveans MR, Taylor M. Does arthroscopic FAl correction improve function with radiographic arthritis? Clin Orthop Relat Res. 2011;469:1667-76. doi:10.1007/s11999-010-1741-6.

23. Moseley JB, O'Malley K, Petersen NJ, Menke TJ, Brody BA, Kuykendall DH, Hollingsworth JC, Ashton CM, Wray NP. A controlled trial of arthroscopic surgery for osteoarthritis of the knee. N Engl J Med. 2002;347:81-8. doi:10.1056/NEJMoa013259.

24. Lodhia P, Slobogean GP, Noonan VK, Gilbart MK. Patient-reported outcome instruments for femoroacetabular impingement and hip labral pathology: a systematic review of the clinimetric evidence. Arthroscopy. 2011;27:279-86. doi:10.1016/j.arthro.2010.08.002.

25. Spencer-Gardner L, Eischen JJ, Levy BA, Sierra RJ, Engasser WM, Krych AJ. A comprehensive five-phase rehabilitation programme after hip arthroscopy for femoroacetabular impingement. Knee Surg Sports Traumatol Arthrosc. 2014;22:848-59. doi:10.1007/s00167-013-2664-z.

26. Tannast M, Siebenrock KA, Anderson SE. Femoroacetabular impingement: radiographic diagnosis-what the radiologist should know. AJR Am J Roentgenol. 2007;188:1540-52. doi:10.2214/AJR.06.0921.

27. Chakraverty JK, Sullivan C, Gan C, Narayanaswamy S, Kamath S. Cam and pincer femoroacetabular impingement: $\mathrm{CT}$ findings of features resembling femoroacetabular impingement in a young population without symptoms. AJR Am J Roentgenol. 2013;200:389-95. doi:10.2214/AJR.12.8546.

28. Register B, Pennock AT, Ho CP, Strickland CD, Lawand A, Philippon MJ. Prevalence of abnormal hip findings in asymptomatic participants: a prospective, blinded study. Am J Sports Med. 2012;40:2720-4. doi:10.1177/ 0363546512462124.

29. Rubin DA. Femoroacetabular impingement: fact, fiction, or fantasy? AJR Am J Roentgenol. 2013;201:526-34. doi:10.2214/AJR.13.10913.

30. Monazzam S, Bomar JD, Dwek JR, Hosalkar HS, Pennock AT. Development and prevalence of femoroacetabular impingement-associated morphology in a paediatric and adolescent population: a CT study of 225 patients. Bone Joint J. 2013;95-B:598-604. doi:10.1302/0301-620X.95B5.30118.

31. Beaule PE, Hynes K, Parker G, Kemp KA. Can the alpha angle assessment of cam impingement predict acetabular cartilage delamination? Clin Orthop Relat Res. 2012;470:3361-7. doi:10.1007/s11999-012-2601-3.

32. Laborie LB, Lehmann TG, Engesaeter IO, Engesaeter LB, Rosendahl K. Is a positive femoroacetabular impingement test a common finding in healthy young adults? Clin Orthop Relat Res. 2013;471:2267-77. doi:10.1007/s11999013-2850-9.

33. Troelsen A, Mechlenburg I, Gelineck J, Bolvig L, Jacobsen S, Soballe K. What is the role of clinical tests and ultrasound in acetabular labral tear diagnostics? Acta Orthop. 2009;80:314-8. doi:10.3109/17453670902988402.

34. Ayeni O, Chu R, Hetaimish B, Nur L, Simunovic N, Farrokhyar F, Bedi A, Bhandari M. A painful squat test provides limited diagnostic utility in CAMtype femoroacetabular impingement. Knee Surg Sports Traumatol Arthrosc. 2014;22:806-11. doi:10.1007/s00167-013-2668-8.

\section{Submit your next manuscript to BioMed Central and we will help you at every step:}

- We accept pre-submission inquiries

- Our selector tool helps you to find the most relevant journal

- We provide round the clock customer support

- Convenient online submission

- Thorough peer review

- Inclusion in PubMed and all major indexing services

- Maximum visibility for your research

Submit your manuscript at www.biomedcentral.com/submit
( ) BioMed Central 